\begin{tabular}{|c|c|}
\hline \multirow{3}{*}{ 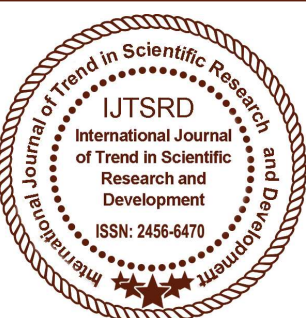 } & $\begin{array}{l}\text { International Journal of Trend in Scientific } \\
\text { Research and Development (IJTSRD) }\end{array}$ \\
\hline & International Open Access Journal \\
\hline & ISSN No: 2456 - 6470 | www.ijtsrd.com | Volume - 2 | Issue -3 \\
\hline
\end{tabular}

\title{
Secure101: Automatic Surveillance and Information Security
}

\author{
Sahil Sharma \\ Department of Information \\ Technology, HMR Institute \\ of Technology, New Delhi
}

\author{
Sneh Rathore \\ Department of Information \\ Technology, HMR Institute \\ of Technology, New Delhi
}

\author{
Lisha Singh \\ Assistant Professor, Dept \\ of Information Technology, \\ HMR Institute of \\ Technology, New Delhi
}

\section{ABSTRACT}

Secure101 is a Python based project, developed for the implementation of the concepts related to Digital Image Processing for Automatic Video Surveillance and Information Security. These are the basic features that have been provided in this project, named as Automatic Surveillance and PiCrypt. The primary objective of this project is to implement the Image Processing Technique by using the available resources like Camera and a Mouse or any motion generation device to construct a single platform that provides the user with some Security tools. To express the features, Python has been selected as an interacting language. For the ease of a user, GUI has been provided for their usage. Though the GUI has been operated and guided by Python script, but there is no need for a person to know the language, for general usage.

Keywords: digital image processing, Automatic Surveillance, PiCrypt, GUI, Information security

\section{INTRODUCTION}

"Secure101" is an intelligent security system combining together the advantages of video surveillance, home security automation, an alarm system, and Image Steganography using dynamic key generation. The fundamental unit of the complete system is based on the implementation of the electronic components of a personal computer (PC) that supports cameras and motion generating and tracking devices such as mouse and touchpad. All this is combined together using the Python platform for Digital Image Processing. This system is a complete solution to various applications, like, security against illegitimate or unauthorized intrusions, safeguard the personal belongings using alert management, monitor and record all the changes in the surrounding, hide and protect secret information. As security has always been one of the biggest concern both for and individual as well as for any organisation. The need for such an application is extensively increasing. The two security modules- automatic surveillance and PiCrypt work independently to help provide the above listed functionalities. The module Automatic surveillance can be utilized both inside as well as outside the premises to increase security aspects of the houses, offices, private apartments, hospitals, shops, as well as in backyards, gardens, parking spaces, building surroundings, especially near entrances or windows. This module is based on visual content analysis algorithms (VCA). These algorithms are designed in such a manner that it maximizes the efficiency of the application. Further to this, it can also be used in the field of Information security which is the second module of this application, named as PiCrypt. It allows a user to hide the secret information within an image with the help of dynamic key generation. The key used for hiding the data is based on the coordinates that are tracked within the application under motion tracking framework. This enhances the security of the data within an image and hides its existence from others. It can be utilized for at both personal and organizational level.

Both Video Surveillance and Information Security have always been an active topic for research and development. For Steganography, various methods and algorithms are available for hiding the information within an image. But the risk of 
information being leaked due to the generalized use of such algorithms make this process less secure. In order to ensure the security and safety of the secret information being hidden within the image, dynamic keys and technique should be used. This makes it difficult for the intruders and for the untrusted parties to fetch out the data from image. The major parts of this project are-

- To provide a tool to automate the monitoring of the footage coming from the camera source.

- To sense the intrusion in the video footage by using techniques like motion detection and object tracking.

- To provide a tool that can secretly hide the information inside an image to make it unsusceptible for the intruder.

- To generate an alert in case of some threat is detected so that quick actions can be taken by the authorities. These alerts can be in the form of emails and SMS.

\section{HARDWARE AND REQUIREMENTS}

SOFTWARE

\section{Software Specification}

- Platform -Python (Version 2 and above)

- GUI Designing - Tkinter

- Computer Vision - OpenCV (Version 2 and above)

- Alert Generation - 1. SMTP Client Package, MIME (For E-mail)

- Twilio (For SMS)

- Graph Plot and Presentation - Bokeh

- Image Analysis - Pillow (PIL)

- Operating System-Windows/Linux/MAC

- More Packages and Libraries

$\circ$ Multiprocessing
$\circ$ Numpy
$\circ$ Pandas

\section{Hardware Specification}

- Web Camera

- Memory: 2GB RAM

- Secondary Storage: 20 GB Hard disk

- Processing Power: Intel Dual core processor

- Mouse or a Touch Panel

\section{WORKFLOW}

The Secure101 application developed here is for the use of providing a low cost with high quality security assurance and alert management from intruders and other untrusted parties. Secure101 is divided into two modules that relate to security in general. The modules are: Automatic Surveillance and PiCrypt. Both these modules works independently and does not depend on the functionality of each other. Automatic Surveillance makes use of the system's camera and a display module for real time video monitoring. PiCrypt uses the user's mouse for generating a key storing the coordinates of the path taken by the mouse on the screen, then secret message is stored in these coordinates using the encoding module by modifying the pixel values of these positions. As a result the user get a Image with message behind the pixels.

Further to make the application easily available to all, an executable file has been created. The .exe file is to be installed in the system and thus can be used for required purpose. The advantage of the software is the independent platform. No Restriction to OS. The use of python makes the developers more comfortable, as the open community language for it, makes the project worthier. The Graphical User Interface here used is tkinter.

\section{FLOWCHART}

The flow of control begins with the initiation of the application, Secure 101. Here the user is provided with two services-

1. Automatic Surveillance

2. PiCrypt(Dynamic Key Generation for Hiding Data within Image)

Based on the option chosen by the user, the application performs accordingly. If the service chosen is Automatic Surveillance, the following set of processes take place one after the other.

1. First the Display Module is called using the Computer Vision where Real Time frames are monitored and processed.

2. As soon as some intrusion is encountered, alerts are generated in the form of Emails, SMS, and graphs.

When the user selects PiCrypt as the working service, the following set of processes take place-

1. User is asked to generate a dynamic Key based on the motion tracking system that records the coordinates of the mouse movement over the tracker screen.

2. This key is then used to hide the secret information within a selected image from Secure101.

3. This encoded image can be shared further and decoded using the application only. 
The above explained flow of control can be pictorially depicted as-

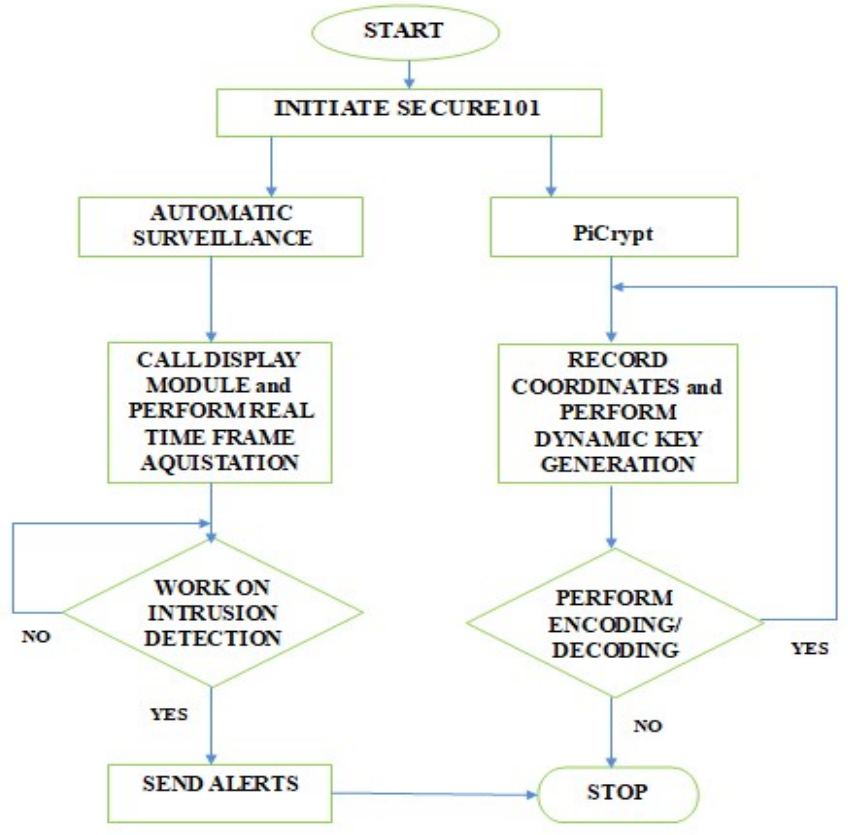

Figure 1 Flow Chart
4. Alert and sharing (Encoded Image): Whenever an intrusion is occurred, then an alert is generated in the form of an email or a SMS. User gets notified about the intruder with the intruder's image and a small video during the time of intrusion.

\section{Saving Records:}

A complete timestamp is recorded for each activity during the intrusion and even during the there is no intrusion. Owner is notified with complete graph showing all the time when there was some activity in the area.

6 Uninstallation: Secure101 can only be uninstalled with the owner's permission. A normal uninstallation is not possible without the permissions.

\section{TECHNICAL IMPLEMENTATION}

\section{Programming Language Used:}

The language used in development of this project is Python. Python is Open Source so we don't need to pay for licensing and python provides rich sets of built in libraries for handling network and web related data in effective and efficient manner. In this project, Python is used for writing scripts and designing algorithms that are required to run the application.

\section{GUI Designing:}

In this project, Tkinter is used for GUI designing. This provides the users of the application fast access to all its features and eases the usability of the security system. Tkinter is the standard GUI library for Python and provides a powerful object-oriented interface to the Tk GUI toolkit.

\section{Smtplib:}

The Alert module of the application covers sending Emails to the owner. These emails include the Video Alerts and the Snapshot alerts. In order to send the emails spontaneously while the program is under running phase, smtplib is used. This module forms a SMTP client session object which is used to send mail to some Internet supported system with an SMTP listener daemon.

\section{OpenCV:}

In this project, OpenCV (Open Source Computer Vision) is used for detection of objects in the video frames being received from the camera source. OpenCV provide feature for detecting faces in the image and provide cascade classifiers to classify and 
recognize the Face in the Image. This project is using openCV to train the dataset with the owner's image, to authorize or unauthorized the person in the image or frame by comparing the owner's dataset with the person's image feature. It matches the feature of current frame with the predefined dataset. OpenCV also provide tools to read, resize the frame as per the analysis requirement of the project.

\section{PIL:}

Python Imaging Library also known as Pillow is a library for the Python programming language which is implemented in this project for adding support for opening, manipulating and saving files that are present in image file format. Here, in this project, PIL is used for manipulating the image file format by hiding the secret information within an image and later for decoding the information.

\section{Twilio:}

The alert module also includes the sending and receiving of SMS on the owner's registered mobile number. Using Python, sending of SMS while the script is running is done using Twilio. This allows sending SMS within the programming control. Here in this project, Twilio is used to send the Alert SMS to the owner, warning against some intrusion conducted in his absence.

\section{SOFTWARE IMPLEMENTATION}

Secure101 is software which is implemented as a Desktop application to provide two features: Automatic Surveillance and PiCrypt. The GUI is designed using the Tkinter, which is a python based tool for designing interfaces. Secure 101 has two modules that work independently of each other. These modules are called from a common interface to provide the functionality to the user.

First module is for Automatic surveillance, which calls a display module which shows the current view of the camera and gives current frame to the analyzer to analyze the video frame by frame. This module then applies techniques of Face Detection and Face Recognition to detect any kind of intrusion.

Second module is for PiCrypt which is an image steganography tool to hide sensitive information behind an image without anyone knowing. It generates the key dynamically by recording the user's mouse movements in real-time. Then the user's message is stored in the pixels depicted by the key.

These two modules are combined as a whole to provide general security feature using Digital Image Processing.

Software is implemented as a Desktop application with the help of scripts. Application is called using the main script which imports the required packages and loads the Interface. All the modules are accessible through the main script. The application is compatible with all the Operating Systems i.e. Linux, Windows, Mac OS etc.

\section{SCREENSHOTS}

The application Secure101 begins as-

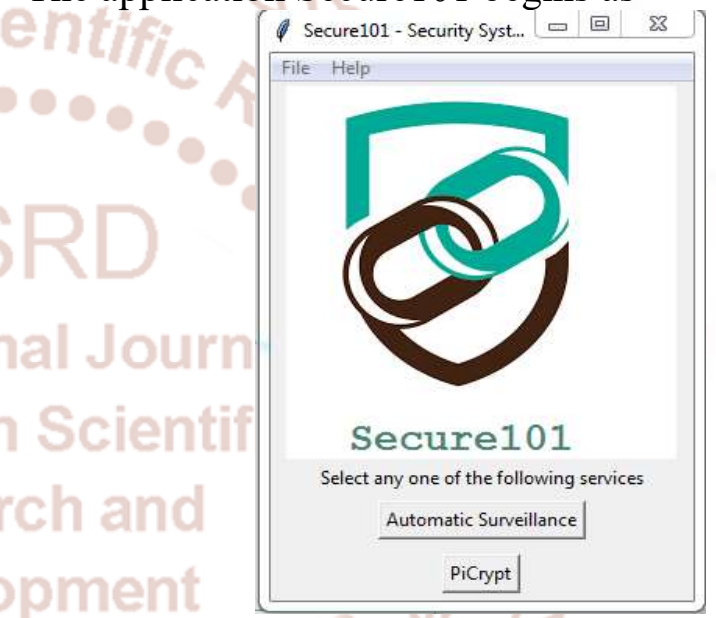

Figure 2 Secure101 Security Systems

Here the user is provided with two choices-

1. Automatic Surveillance

2. PiCrypt

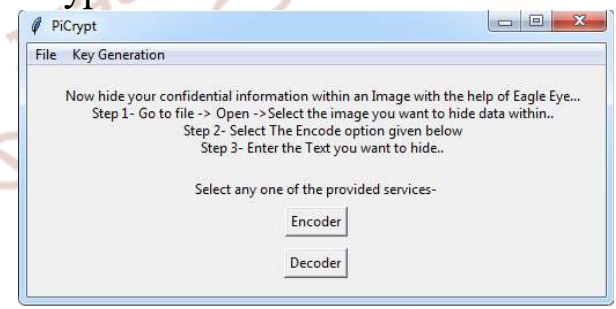

Figure 3 PiCrypt Module

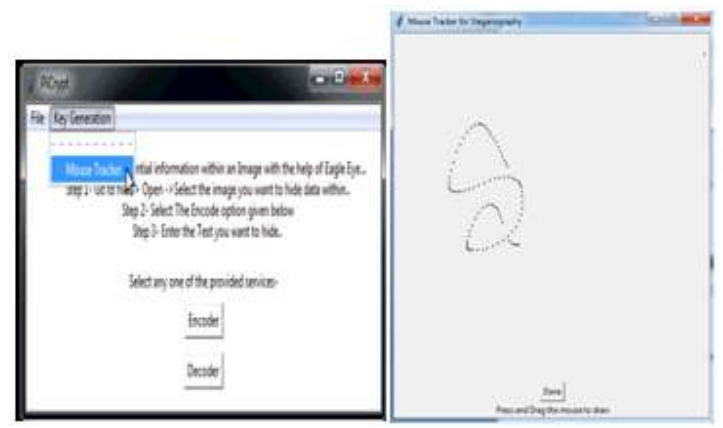

Figure 4 Generation of Key 


\section{CONCLUSION \& FUTURE SCOPE}

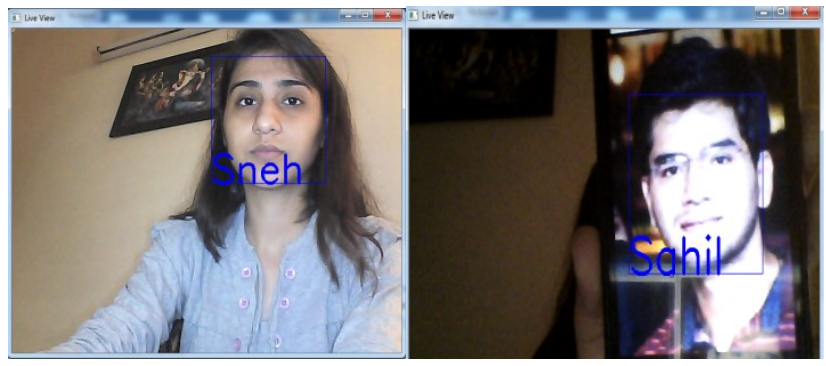

Figure 5(a, b) Recognized Faces

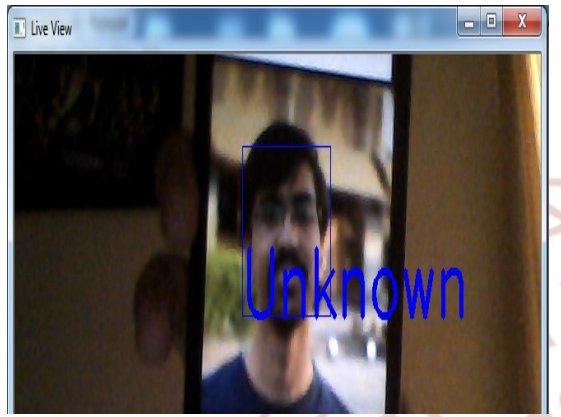

Figure 6 Un-Recognized Face addressed as unknown

On the basis of the intrusion detection alerts are generated as-

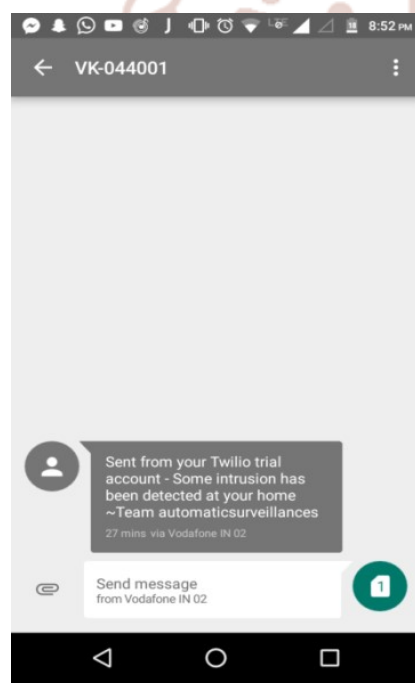

Figure 7 SMS Alert

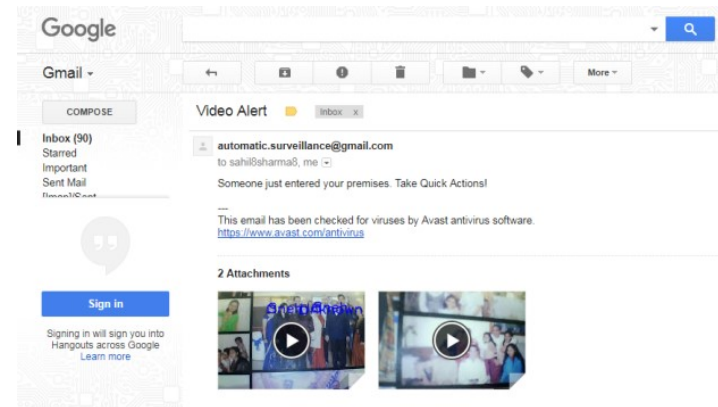

Figure 8 E-mail Alert
In current work we developed the system that provides security tool using Digital Image Processing for automatic surveillance and information security. This system is developed in python and has an interactive GUI for the ease of the user. The Data, which can be protected with the help of the module named as PiCrypt which is evolved from Information Security using Steganography. The usage of traditional techniques and algorithms make the data hiding process less secure. In order to avoid this, we have used the approach of Dynamic Key Generation by recording the coordinates of the mouse or any touch panel and further used for hiding data within the image. The second service, that is, Automatic Surveillance helps the user to protect the belongings even in his absence. With the help of this, the installation of CCTV cameras within a home, office or anywhere can be deprecated. In this way, the need of continuous monitoring of the environment or rewinding of the recorded video when some mishappening has been conducted is replaced by the Alert Management module of the application. The user receives emails, SMS and graph whenever some unwanted disruptions are encountered which helps the owner to take specific actions right in the nick of time.

Thus the provided features in the above discussed software give it long scope in the future as-

1. Implementation of the application on Raspberry Pie to make it portable and flexible for usage.

2. Live Coverage of the environment under surveillance on the mobile phone of the owner. This requires the development of the mobile app.

3. The application can be used for monitoring ill people and track their movements.

4. The Surveillance module can be implemented for traffic management.

5. The PiCrypt can further be extended to image analysis system where the details of a particular image in terms of the visual features made available in text form.

Further to these there are many more aspects where the development of the application can be extended. 


\section{REFERENCES}

\section{Website References}

1. https://docs.opencv.org/3.0-

beta/doc/py_tutorials/py_tutorials.html

2. https://link.springer.com/chapter/10.1007/978-1-

4615-4375-6_2

3. opencv-python-

tutroals.readthedocs.io/en/latest/...tutorials/.../py_i

mage_display.html

4. https://www.pyimagesearch.com/

5. https://www.pyimagesearch.com/2017/08/14/long -exposure-with-opencv-and-python/

\section{Book References:}

1. Al Sweigart - Automate the boring Stuff with Python, First Edition

2. Joseph Howse, Prateek Joshi -OpenCV: Computer Vision Projects with Python, First Edition.

3. J. M. Hughes - OpenCV: Computer Vision Projects with Python, 2nd Edition.

4. Adrian Rosebrock - Practical Python and OpenCV, 3rd edition. 